\title{
Muss das Spital der Zukunft mehr sein als nur ein Spital?
}

\author{
Kooperationen zwischen Arztpraxen, Spitälern oder Pflegeheimen haben wegen \\ der potentiellen Kostenreduktion durch Synergieeffekte Auftrieb erhalten. Kann die \\ engere Anbindung von Praxen an ein Spital unter dem Gesichtspunkt Managed \\ Care längerfristig von Vorteil sein?
}

Michael Zaugga, Jörg Kündigb, Andreas Gattikerc

a Professor of Anesthesiology, University of Alberta, School of Medicine and Dentistry, Canada

b Verwaltungsratspräsident der GZO Spital Wetzikon, Kantonsrat FDP ZH

c Vorsitzender der Geschäftsleitung der GZO Spital Wetzikon

Korrespondenz:

Dr. Andreas Gattiker GZO AG

Spital Wetzikon

Spitalstrasse 66

CH-8620 Wetzikon

Tel. 0449342701

direktion[at]gzo.ch

\section{Aktuelle Situation im \\ schweizerischen Gesundheitswesen}

Verschiedene ökonomische Umwälzungen im Schweizer Gesundheitswesen füllen täglich die Zeitungsspalten. Am prominentesten erscheint sicher die leistungsbezogene DRG-basierte Spitalfinanzierung, die seit dem 1. Januar 2012 in Kraft ist. Sie soll zu mehr Wettbewerb im Spitalbereich und je nach Autor zu Kostensenkungen oder einem verminderten Kostenanstieg führen. Aktuell ist in diesem Bereich noch vieles unklar, vor allem die Widersprüche zwischen dem Art. 49 KVG und dem Art. 59 KVV blockieren die Entfaltung der politisch beabsichtigten Intention. Aber auch die unter dem Etikett der gemeinwirtschaftlichen Leistungen (GWL), die von den Kantonen unter verschiedensten Begründungen an «ihre Spitäler» ausgeschüttet werden, führen zu Marktverzerrungen und verhindern letztlich die notwendige Bereinigung im Spitalbereich.

In diesem neuen Umfeld spielen neben der neuen Spitalfinanzierung Kooperationen, Netzwerke und Allianzen (Managed Care) zwischen einzelnen Leistungserbringern (Arztpraxen, Spitälern, Pflegeheimen) eine ebenso wichtige Rolle wie die Veränderungen im Spitalbereich selbst. Beide Neuerungen, die Spitalfinanzierung und Managed-Care-Allianzen führen trotz der oben erwähnten Marktverzerrungen zu einer vermehrten Konsolidierung im Gesundheitsmarkt. Diese umfasst neben Schliessungen und Fusionen von Spitälern auch eine zunehmende Optimierung über die Wertschöpfungskette hinweg. Bereits seit Jahren nimmt die Anzahl der Einzelarztpraxen ab, während die Zahl der Gruppenpraxen zunimmt. Waren 2002 noch über 68\% der Ärzte in Einzelpraxen tätig, so sind es 2011 nur noch knapp 62\% [1]. Der ökonomische Treiber dieser Entwicklung ist die klassische Skalenökonomie bei der Auslastung der kapitalintensiven Praxisinfrastruktur, verstärkt durch zunehmende Teilzeittätigkeiten der Ärzteschaft in den Praxen. Neben dieser internen Praxiskonsolidierung kommen seit Anfang der 1990er Jahre auch externe Player ins Spiel. So eröffneten

\section{L'hôpital du futur doit-il être plus qu’un hôpital?}

Le nouveau financement hospitalier s'accompagne de nombreux bouleversements économiques. Outre les changements dans le domaine hospitalier, les exemples de coopérations entre les fournisseurs de prestations (cabinets médicaux, hôpitaux, établissements de soins) se multiplient, notamment en raison des économies potentielles qu'elles permettent de réaliser. Le rachat de cabinets par les assureurs et autres investisseurs privés propriétaires de «chaînes de cabinets» vient également renforcer cette tendance à la consolidation. Le présent article tente d'analyser les lois économiques qui régissent cette évolution et de les replacer dans le contexte international. Cette analyse stratégique a notamment révélé que l'intégration étroite de cabinets médicaux dans un hôpital peut, à long terme, s'avérer intéressant pour tous les acteurs concernés (médecins, hôpitaux, patients et répondants des coûts), selon le principe du Managed care. L'émergence de monopoles et d'une distorsion de la concurrence est peu probable en Suisse en raison de la fragmentation du marché de la santé. Néanmoins, il convient de surveiller attentivement certains risques potentiels comme l'endettement et les conflits culturels.

einige Versicherungen HMO-Praxen, um so bereits zu Beginn des Behandlungsprozesses auf die Kosten Einfluss zu nehmen. Mit dem Praxisstopp ab 2002 erwartete die Politik mit der Argumentation «Jeder zusätzliche über die Grundversicherung abrech- 
nende Arzt kostet diese eine halbe Million» Einsparungen bei den Gesundheitskosten. Wie so oft trat der politisch erhoffte Effekt nicht ein. Während des Zulassungsstopps stieg die Zahl der Ärzte gleich schnell wie davor, und auch die Kosten für ambulante Leistungen stiegen unvermindert weiter. $\mathrm{Zu}$ sätzlich wurden Praxisbewilligungen plötzlich zu einem knappen und dadurch handelbaren Gut, das bei einer Praxisübernahme ebenfalls rentabilisiert werden musste. Die Übernahme einer Praxisbewilligung verbunden mit dem Kauf einer oftmals veralteten Praxis erschien angesichts dieser schlechten Einkommensaussichten (TARMED) nicht mehr interessant. In die Lücke sprangen in den letzten Jahren zunehmend Anbieter, die Praxen aufkauften, diese in einer Kette betrieben und die Ärzte im Anstellungsverhältnis beschäftigten. Bekanntestes Beispiel ist sicher die Firma santémed, unter der die Swica ein Netz von Praxen in Städten und grösseren Gemeinden betreibt, aber auch Private, unter anderem die Migros. Unter solchen Rahmenbedingungen lohnt es sich, die Frage zu klären, inwiefern Spitäler, die von einem funktionierenden Praxisumfeld in ihrer Region abhängig sind, bei der anstehenden Konsolidierung eine Rolle übernehmen können oder sollen.

\section{GZO Spital Wetzikon bildet regionale Praxiskette}

Auch Spitäler sind auf eine gute regionale Hausarztversorgung angewiesen. Daher hat die GZO Spital Wetzikon mit der GZO Partner AG eine Tochterfirma gegründet mit dem Zweck, junge Ärzte beim Einstieg in die Praxistätigkeit zu unterstützen und das persönliche Investitionsrisiko für diese zu senken.

Die GZO ist überzeugt, dass ein öffentliches Spital als Leistungserbringer ein natürlicher Partner für die Sicherstellung der Grundversorgung sein kann.

Im Folgenden wird das Potential einer solchen «Zentralisierung der Versorgungskette» kurz diskutiert.

\section{Ökonomie der vertikalen Integration}

Vertikale Integration ist per definitionem das Zusammenlegen von zwei Firmen (im vorliegenden Fall Spital + Arztpraxis), die im Produktionsprozess, respektive der Serviceleistung, auf unterschiedlicher Höhe der Wertschöpfungskette (Therapie, Heilung, Rehabilitation) agieren (s. Abb.). Die Lehre der Öko-

\section{Abbildung 1}

Beim Zukauf von Arztpraxen ( $P$ ) ins Spitalgeschäft $(H)$ handelt es sich um eine zentralisierte Versorgungskette mit vertikaler Rückwärts(upstream)- und Vorwärts(downstream)-Integration. Die Zuweisung von Patienten ist bidirektional.

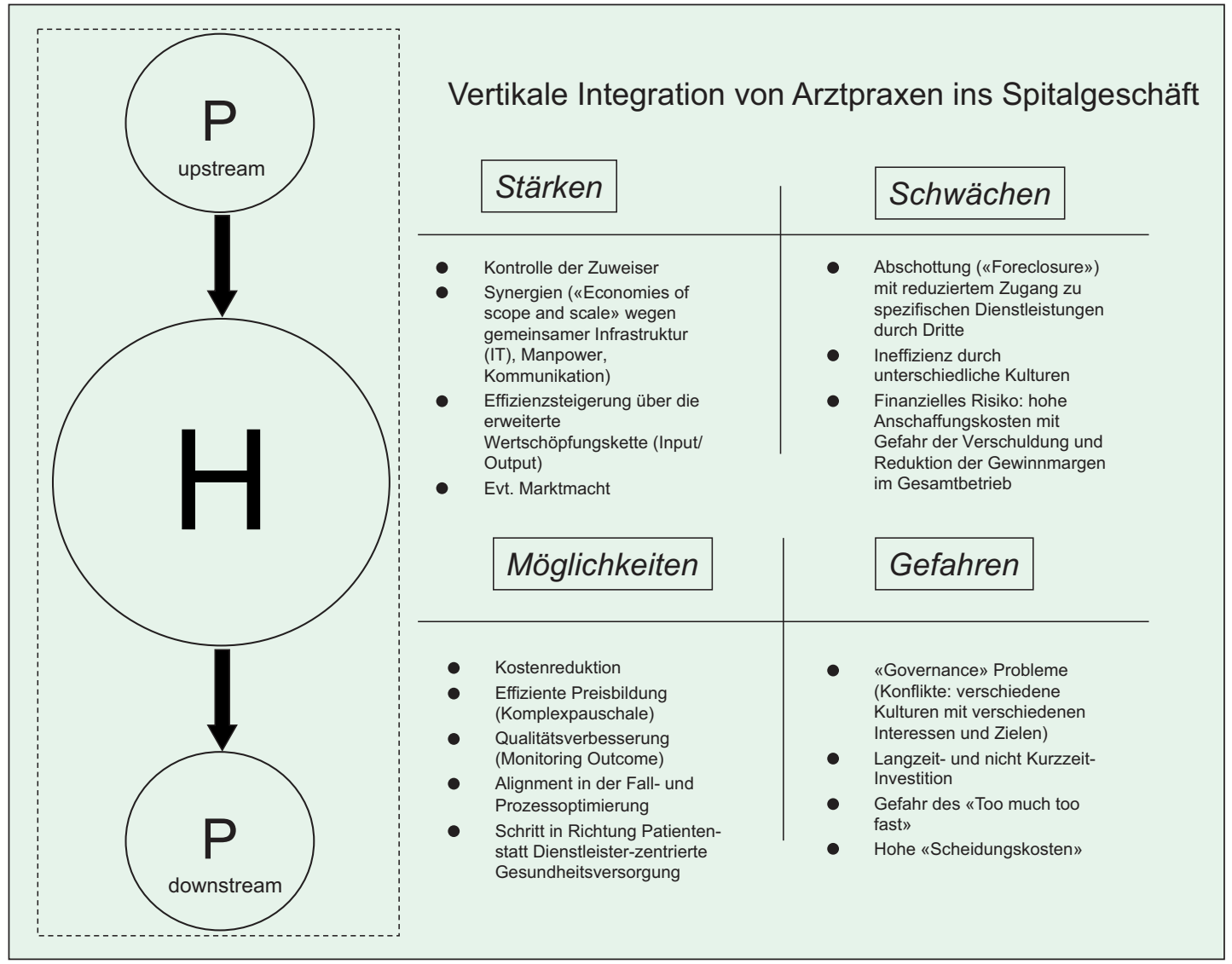




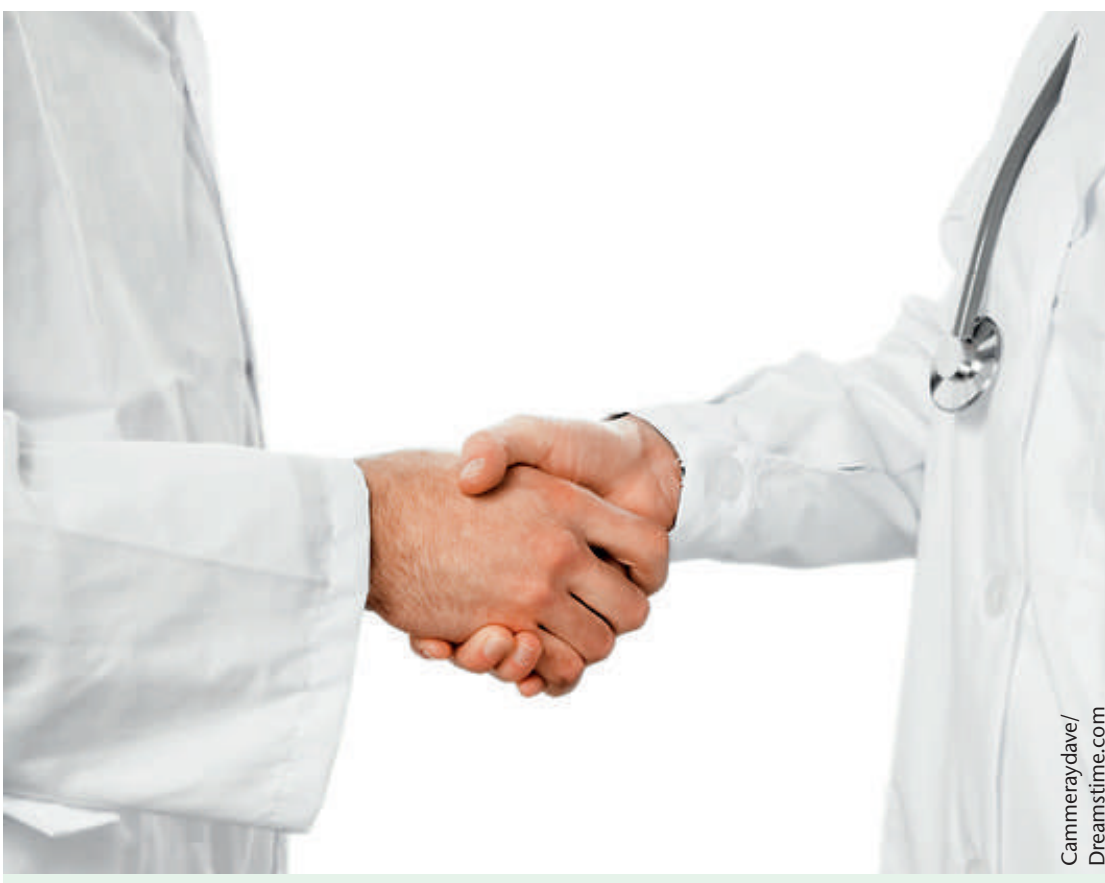

Kann die Zusammenarbeit zwischen Einzelpraxis und Spital für beide Seiten von Vorteil sein?

nomie besagt, dass vertikale Integration sich je nach Model effizienzsteigernd, aber auch wettbewerbsverzerrend (anti-kompetitiv) auswirken kann [2]. Die Effizienzsteigerung wird durch Sicherung der Patientenzufuhr und gleichzeitiger Senkung von Trans-

\section{Ist der Zukauf von Arztpraxen durch Spitäler nun effizienzfördernd oder -verhindernd?}

aktionskosten erzielt. Die Zusammenlegung der «Profitabilitätsrechnungen» zweier Firmen führt zudem zu niedrigeren Preisen, bei gleichzeitig grösseren Margen und höherer Profitabilität (Konsolidierte Effizienzsteigerung). Andererseits kann eine starke Reduktion unabhängiger Arztpraxen im Einzugsgebiet eines Spitals zu einer Abschottung oder im Extremfall zu einer Kartellbildung führen, was eine Zunahme der (regionalen) Marktmacht der Spitäler gegenüber Versicherern und höhere Preise nach sich ziehen würde. Die Effekte von Effizienz und Marktmacht auf die Preisbildung heben sich im Regelfall jedoch auf. Im stark fragmentierten Schweizer Spital- und Praxismarkt ist der Aufbau einer Marktmacht sehr unwahrscheinlich. Auch unterliegen die Preise im Gesundheitswesen nicht vollständig den Marktkräften, sondern werden weitgehend politisch aufgrund von Kostenrechnungen bestimmt. Das erschwert eine monopolistisch geprägte Preisbildung ebenfalls.

\section{Vertikale Integrationsaktivitäten in anderen Gesundheitssystemen}

Die vorhandene Literatur zur vertikalen Integration von Arztpraxen ins Spitalgeschäft ist verglichen mit der Literatur zur horizontalen Integration (z. B. Spitalfusionen) im Gesundheitswesen eher bescheiden und ziemlich kontrovers. Die meisten Studien über dieses Thema wurden in den 90er Jahren im amerikanischen Gesundheitswesen durchgeführt. Damals hat sich als Folge der Einführung von DRGs und dem aufblühenden Managed-Care-Konzept ein regelrechter Boom entwickelt [3]. Viele Spitäler verfolgten eine aggressive Expansionspolitik, im Glauben, als sogenannte «First Mover» einen Wettbewerbsvorteil zu erlangen, eine Rechnung, die aber oftmals nicht aufging (Leading - Speeding - Bleeding). Neben dem eigentlichen Kauf von Arztpraxen durch private Ketten (HMO) und Spitäler (Integrated Service Model) haben sich auch andere weniger feste Formen der Zusammenarbeit (Independent Practice Associations oder Physician Hospital Organizations) etabliert [4]. 1996 hatten ca. 40\% aller US-Spitäler strategische Allianzen mit Arztpraxen, ein Prozentsatz, der sich aber über die Zeit wieder reduziert hat und heute bei etwa $25 \%$ liegt.

Die Ursachen für dieses Gesundschrumpfen sind vielschichtiger Art. Sicher spielen zu grosse Erwartungen von Seiten der Spitäler und das Aufeinanderprallen von verschiedenen Kulturen (Praxis vs. Spital) eine wichtige Rolle, ähnlich wie bei den eher wenig erfolgreichen Spital-gesponserten HMOs. Die Spitäler standen zunehmend auch in der Kritik der Regulierer und Versicherer, die eine Verzerrung des freien Marktes durch Kartellbildung befürchteten und dadurch eine weitere Zunahme der Kosten im Gesundheitswesen [5]. Zusätzlich musste man auch erfahren, dass die Kosten einer allfälligen späteren Trennung oft sehr hoch waren (Gerichtskosten). Auf dem Hintergrund dieser Erfahrungen stellt sich die Frage, ob der Zukauf von Arztpraxen durch Spitäler nun effizienzfördernd oder -verhindernd ist? Mehrere Studien konnten keine finanzielle Verbesserung bei Spitälern mit Integration von Arztpraxen nachweisen [6]. Im Gegensatz dazu konnte aber eine Studie aus Kalifornien (2001) mit 363 Akutspitälern höhere Margen und einen höheren ROA (return on assets) bei denjenigen Spitälern zeigen, die Arztpraxen in ihr Geschäftsmodell integriert hatten [7]. Bei der vertikalen Integration zwischen Akutspitälern und Langzeitpflegeheimen erwartete man eine verbesserte Effizienz durch Kostensenkungen in der Gesamtbehandlung. Entgegen dieser Erwartung verschlechterten sich aber die Gewinnmargen. Ob dies durch Probleme bei den Schnittstellenkosten oder aber durch zu aggressive Preisnachlässe bei den kombinierten Pauschalpreisen (Komplexpauschalen) bedingt war, wurde hier nicht eruiert. Auch in Deutschland wird seit dem Jahre 2000 das Konzept der Integrated Primary Care aggressiv vorangetrieben 
[8]. Dieses stützt sich bisher mehr auf Kooperation und Koordination als auf den eigentlichen Erwerb von Arztpraxen, obwohl ein solcher von der deutschen Gesetzgebung her möglich wäre. Es scheint, dass sich in solchen Kooperationen insbesondere ein günstiger Effekt auf die Qualität der Dienstleistung und den Patienten-Outcome bemerkbar macht. Allerdings zeigen andere (vor allem amerikanische) Studien, dass bei vertikalen Integrationsaktivitäten eher ein Preisanstieg bei ambulanten Leistungen als eine verbesserte Qualität resultiert. Die Ursache für die Preissteigerung ist in diesen Fällen in der Monopolisierung des Marktes zu suchen [9].

\section{Strategische Analyse}

Um das Potential der Integration von Arztpraxen ins Spitalgeschäft abzuschätzen, wurde basierend auf der vorhandenen Literatur eine SWOT(strengths, weaknesses, opportunities, threads)-Matrix erstellt (s. Abb.). Diese zeigt zusammenfassend Folgendes: Im Vordergrund stehen die Möglichkeit der Kostenreduktion durch Synergien (economies of scope and scale), Prozessoptimierung und Qualitätsverbesserung. Aus Sicht der Spitäler kann die vertikale Integration attraktiv sein, um einerseits die regionale Versorgung sicherzustellen und Einfluss am Beginn der Behandlungskette zu haben. Auch die Entlastung der Notfallstation als sehr teure Anlaufstelle ist nicht zu unterschätzen. Auch für junge Ärzte kann dieses Modell von Vorteil sein, da ein finanzstarker Partner der Behandlungskette als Kapitalgeber fungiert. Wichtige Erfolgsfaktoren sind aber hierbei (1.) der richtige Standort, (2.) die Fähigkeit, eine Praxis personell auch auszulasten, (3.) die Umsetzung einer unternehmerischen Kultur und nicht zuletzt (4.) auch interessante finanzielle Beteiligungsmodelle für die in den Praxen tätigen Schlüsselpersonen.

\section{Schlussfolgerungen}

Es ist wichtig zu erkennen, dass der Zukauf von Arztpraxen als Teil des Geschäftsmodells eines Spitals interessant sein kann. Die vertikale Integration von Arztpraxen ins Spitalgeschäft muss aber als Langzeitinvestition betrachtet werden, die auch beträchtliche Risiken in Bezug auf Verschuldung, Kulturkonflikte und mögliche Trennungskosten birgt. Während Beispiele für die vertikale Integration aus der Fastfood-Industrie und dem Ölgeschäft deutliche
Vorteile bezüglich Profitabilität zeigen, sind diese im Gesundheitswesen noch kontrovers. Die Gefahr der Abschottung und Kartellbildung besteht in der Schweiz angesichts des fragmentierten Leistungserbringermarktes kaum, sollte aber monitorisiert werden. Allenfalls wird auch die Politik als Folge der unabhängig von der vertikalen Integration weitersteigenden Kosten gesetzliche Massnahmen (erneuter Praxisstopp für Spezialisten; Eigentümerregelungen für Arztpraxen) ergreifen, um so die Rahmenbedingungen des Gesundheitswesens «gut gemeint» zu präzisieren. Diese Massnahmen würden letztlich aber nur den Markt weiterverzerren. Es wird sich zeigen, ob eine «virtuelle» Integration, d.h. eine Integration basierend auf agilen und reversiblen strategischen Allianzen, dem eigentlichen Zukauf von Arztpraxen im Sinne der vertikalen Integration mehr Vorteile bietet. Sicher allerdings ist nur, dass das Spital der Zukunft mehr sein muss als nur ein «Spital».

\section{Literatur}

1 FMH-Ärztestatistik. Zahlen und Fakten 2011.

2 Gaynor M. Is vertical integration anticompetitive? Definitely maybe (but that's not final). Journal of Health Economics. 2006; 25:175-80.

3 Burns LR, Pauly MV. Integrated delivery networks: a detour on the road to integrated health care? Health Affairs. 2002;21:128-43.

4 Ciliberto F, Dranove D. The effect of physician-hospital affiliations on hospital prices in California. Journal of Health Economics. 2006;25:29-38.

5 Gaynor M, Haas-Wilson D, Vogt W. Are invisible hands good hands? Moral hazard, competition, and the 2nd best in health care markets. Journal of Political Economy 2000;108:992-1005.

6 Wan TTH, Ma A, Lin BYJ. Integration and the performance of healthcare networks: do integration strategies enhance efficiency, profitability, and image? International Journal of Integrated Care. 2001;1:1-7.

7 Wang BB, Wan TTH, Clement J, Begun J. Managed care, vertical integration strategies and hospital performance. Health Care Management Science 2001;4:181-91.

8 Schlette S, Lisac M, Blum K. Integrated primary care in Germany: the road ahead. International Journal of Integrated Care. 2009;9:1-11.

9 Cuellar AE, Gertler PJ. Strategic integration of hospitals and physicians. Journal of Health Economics. 2006;25:1-28. 